\title{
ATUAÇÃO DO ENFERMEIRO NA ADMINISTRAÇÃO DE MEDICAMENTOS EM UMA INSTITUIÇÃO HOSPITALAR: ESTUDO DESCRITIVO
}

Yaná Tamara Tomasi , Gabriela de Nardi Souza², Julia Valeria de Oliveira Vargas Bitencourt ${ }^{3}$, Alexander Garcia Parker ${ }^{4}$, Jussara Gue Martini5, Joel Rolim Mancia ${ }^{6}$

Pesquisa quantitativa, descritiva, realizada com 33 enfermeiros de um hospital público do Oeste Catarinense. Objetivou descrever a atuação do enfermeiro no processo de administração medicamentosa, evidenciar as potencialidades/fragilidades para inserção prática. Para a coleta de dados, usou-se questionário. Os dados foram tabulados e analisados mediante a análise estatística descritiva. Os enfermeiros identificam, embora parcialmente, seu papel, sendo técnicos/auxiliares os executores da administração medicamentosa. Detecta-se conhecimento, atualização e preparo para a prática, contrariando a uma atuação com escasso envolvimento, justificada pela sobrecarga administrativa/assistencial. Questões éticas e legais são inerentes neste cuidado, enfatizando a discussão da responsabilidade profissional e práticas seguras.

Descritores: Enfermagem, Pesquisa em enfermagem clínica, Sistema de medicação no hospital, Papel do profissional de enfermagem, Responsabilidade legal.

\section{EL PAPEL DE LA ENFERMERA EN ADMINISTRACIÓN DE MEDICAMENTOS EN UN HOSPITAL: ESTUDIO DESCRIPTIVO}

La investigación cuantitativa, descriptiva, realizada con 33 enfermeras de un hospital público de Santa Catarina West.Dirigido para describir el trabajo de las enfermeras en el proceso de gestión de los medicamentos, destacando los potenciales / debilidades de practicar la inserción. Para la recolección de datos se utilizó el cuestionario. Los datos fueron tabulados y analizados mediante estadística descriptiva. Las enfermeras identifican, aunque parcialmente, su papel de ser intérpretes técnicos / auxiliares de la administración del fármaco. Detecta hasta el conocimiento, actualización y preparación para la práctica, en contra de una actuación con poca participación, justificada por la asistencia administrativa / sobrecarga. Aspectos legales y éticos inherentes a este tipo de atención, haciendo hincapié en la discusión de la responsabilidad profesional y las prácticas de seguridad.

Descriptores: Enfermería, Investigación en enfermería clínica, Sistema de medicación en hospital, Rol de la enfermera, responsabilidad legal.

\section{THE ROLE OF THE NURSE IN MEDICATION ADMINISTRATION IN A HOSPITAL: A DESCRIPTIVE STUDY}

Quantitative, descriptive research, carried out with 33 nurses from a public hospital in Santa Catarina West. Aimed to describe the nurse's role in the drug administration process, highlight the potential / weaknesses to practice inclusion. For data collection was used questionnaire. Data were tabulated and analyzed by descriptive statistics.The nurses identify, although partially, its role being technical / auxiliary performers of drug administration. Detects up knowledge, updating and preparation for practice, contrary to a performance with little involvement, justified by the administrative / overhead assistance. Legal and ethical issues inherent in this care, emphasizing the discussion of professional responsibility and safe practices.

Descriptors: Nursing, Clinical Nursing Research Medication systems hospital, Nurse's role, Liability legal.

1Enfermeira. Residente Multiprofissional em Saúde da Familia/Florianópolis. E-mail: yanaa192@hotmail.com.

${ }^{2}$ Aluna do 9ㅇ periodo do curso de Graduação em Enfermagem da UFFS.

${ }^{3}$ Enfermeira. Mestre em Enfermagem. Docente da Universidade Federal da Fronteira Sul- UFFS.

${ }^{4}$ Enfermeiro. Mestre em Enfermagem. Docente da UFFS.

${ }^{5}$ Enfermeira. Doutora em Enfermagem. Docente da Universidade Federal de Santa Catarina- UFSC.

${ }^{6}$ Enfermeiro. Doutor em Enfermagem. Docente da Universidade do Vale dos Sinos-UNISINOS. 


\section{INTRODUÇÃO}

O papel do enfermeiro no processo de trabalho assistencial relativo à terapêutica medicamentosa tem sido foco de questionamentos. Isto ocorre devido ao conhecimento técnico científico relacionado a esta prática e ao distanciamento do enfermeiro, no que tange à supervisão do preparo e administração de medicamentos, em virtude desta responsabilidade muitas vezes ser atribuida ao técnico ou auxiliar de enfermagem ${ }^{(1)}$.

Considera-se que a administração de medicamentos não se configure um cuidado intrinsecamente técnico. Subentende-se um cuidado de extrema complexidade, já que se sobrepõe a esta atividade aspectos emocionais, motivacionais, espirituais, sensibilidade dolorosa e os significados desta administração para o usuário.

A administração medicamentosa é importante prática na enfermagem, de forma que o enfermeiro deve conhecer todos os aspectos envolvidos nesta ação. É atividade que requer conhecimento técnico atualizado, além de visão crítica do profissional(2), tendo em vista que compõe uma habilidade clínica essencial para enfermeiros e sua equipe e acarreta riscos ao usuário e ao próprio profissional(3,4).

O processo do preparo e administração de medicamentos é regido por normas e leis e, apesar destes fatores serem parte essencial na formação de enfermeiros, erros na administração de medicamentos são comuns, principalmente nos hospitais. Indica-se que $\circ$ fato de enfermeiros concentrarem-se em mais de uma tarefa é um fator de risco para ocorrência de erros, visto que a supervisão do processo se torna prejudicada. Jornadas exaustivas são, também, fatores que implicam na qualidade da assistência ${ }^{(2,5)}$

Logo, por meio destas considerações, direcionando ao problema deste estudo, questiona-se: como tem se configurado a atuação do enfermeiro quanto à prática de administração medicamentosa em um hospital público do Oeste Catarinense? A partir deste questionamento, os objetivos são descrever a atuação do enfermeiro no processo de administração de medicamentos em um hospital público do Oeste Catarinense; evidenciar as potencialidades e fragilidades na inserção do enfermeiro nesta prática.

\section{METODOLOGIA}

Trata-se de uma pesquisa descritiva com abordagem quantitativa, autorizado pela instituição pesquisada e aprovado pelo Comitê de Ética e Pesquisa cujo protocolo é: 423.15, desenvolvido nos meses de Dezembro/2013 e Janeiro/ 2014 em um hospital público estadual de referência para o Oeste Catarinense, localizado na cidade de Chapecó. A investigação foi realizada nos seguintes setores: Unidade de Tratamento Intensivo (UTI), Unidade de Tratamento Intensivo Neonatal (UTIN), Clínica Médica Geral, Unidade Cirúrgica, Unidade Ortopédica, Unidade Neurológica, Unidade Oncológica, Unidade Obstétrica e Pronto Socorro (PS). Não foram investigados o Centro Cirúrgico e a Unidade quimioterápica, por se tratarem de setores cuja administração medicamentosa constitui peculiaridade.

Os critérios de inclusão para a pesquisa constituíram enfermeiros assistenciais e administrativos das unidades selecionadas, considerando que estes últimos participam ativamente da gestão da assistência, podendo assim contribuir com a descrição da atuação do enfermeiro acerca desta prática. Foram excluídos do estudo os enfermeiros em período de experiência, ou seja, inferior a 3 meses.

Participaram 33 enfermeiros de um total de 44 das unidades inclusas no estudo; dos 11 profissionais não participantes os motivos foram: o não aceite 8 , férias 2 e afastamento por motivo de doença 1 . O instrumento de pesquisa foi um questionário com perguntas fechadas, aplicado aos enfermeiros após a apresentação dos objetivos do estudo e a assinatura do Termo de Consentimento Livre e Esclarecido.

Os dados foram tabulados com a utilização do programa Excel e analisados mediante estatística descritiva com frequências relativas e ou absolutas ${ }^{(6)}$.

\section{RESULTADOS}

Quanto ao setor de atuação dos 33 participantes do estudo, 7 atuam na UTI; 4 respectivamente na Clínica Médica, UTIN, Neurologia e Obstetrícia; 2 na Cirúrgica; 2 respectivamente na Ortopedia, Oncologia e PS. O tempo de atuação na profissão é entre 3 e 5 anos para 3 participantes, de 1 a 3 anos para 6, até 1 ano para 4 e acima de 5 anos constituindo-se 19 participantes. No turno de trabalho, 13 são do vespertino, 12 do matutino e 12 do noturno. Por fim, a jornada de trabalho é única para 23, dupla para 8 e tripla para 1.

A seguir, demonstram-se os gráficos que elucidam os dados referentes aos objetivos do estudo: 


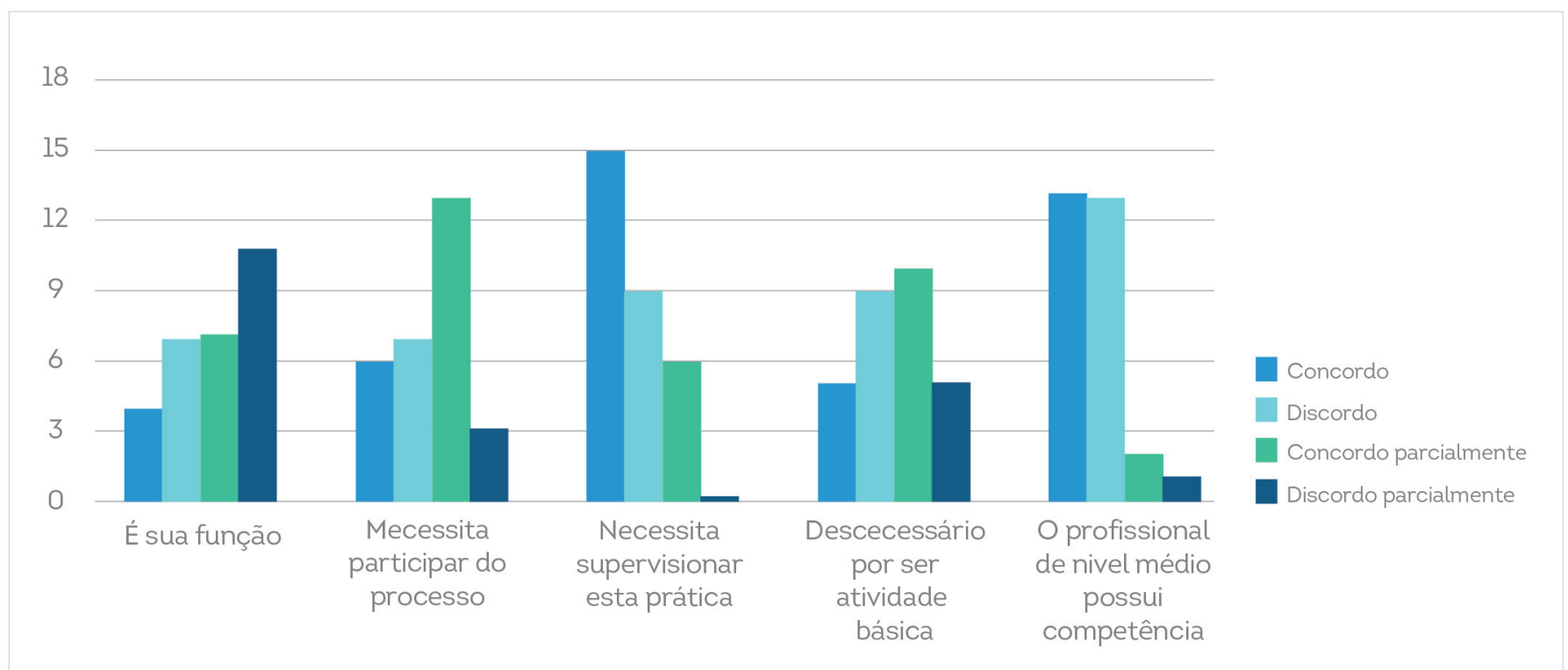

Gráfico 1: Opinião do enfermeiro quanto a sua atuação junto à equipe de enfermagem no processo da administração de medicamentos, Chapecó/SC (2014).

Destaca-se a discordância parcial 11 para a variável que designa se é função do enfermeiro a prática medicamentosa; uma discordância absoluta indicando que o enfermeiro deve participar do processo 13; concordância absoluta afirmando que o enfermeiro necessita supervisionar esta prática 15; valores similares entre a discordância 10 e concordância parcial 9 na afirmação de que a atuação é desnecessária, já que é atividade básica e, por fim, valores iguais para o concordo 13 e o concordo parcialmente 13, ratificando a competência do profissional de nível médio para a execução desta prática.

O medicamento é liberado pela farmácia, conferido, preparado e administrado pelos técnicos/auxiliares e supervisionado pelo enfermeiro sempre que possivel.

O medicamento é liberado pela farmácia, conferido, preparado e administrado pelos técnicos/auxiliares.

O medicamento é liberado pela farmácia, conferido, preparado e administrado pelos técnicos/auxiliares com supervisão contínua do enfermeiro.

O medicamento é liberado pela farmácia, conferido, preparado e administrado pelo enfermeiro.

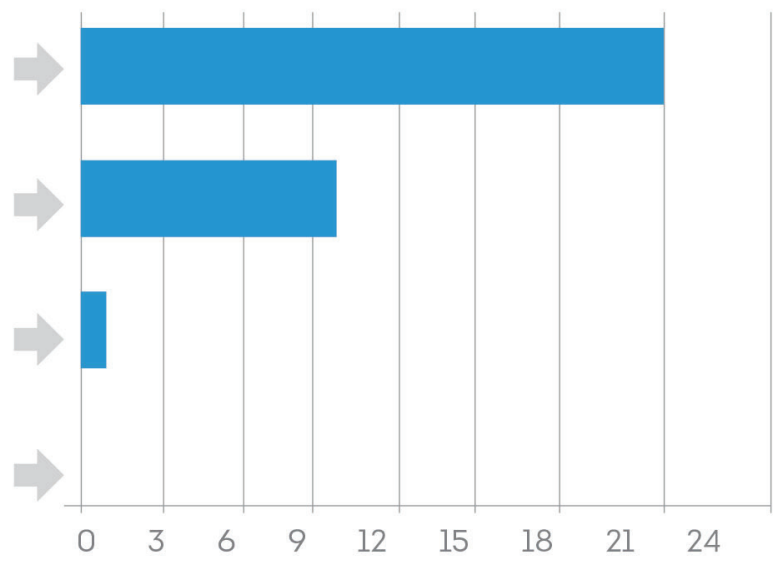

Gráfico 2: Rotinas adotadas desde a conferência do medicamento dispensado da farmácia à administração do medicamento e o papel do enfermeiro, Chapecó/SC (2014).

Enfatiza-se que os enfermeiros indicam que, após a dispensação do medicamento pela farmácia, a supervisão da administração é realizada sempre que possivel e ausência da exclusividade desta prática pelo enfermeiro. 


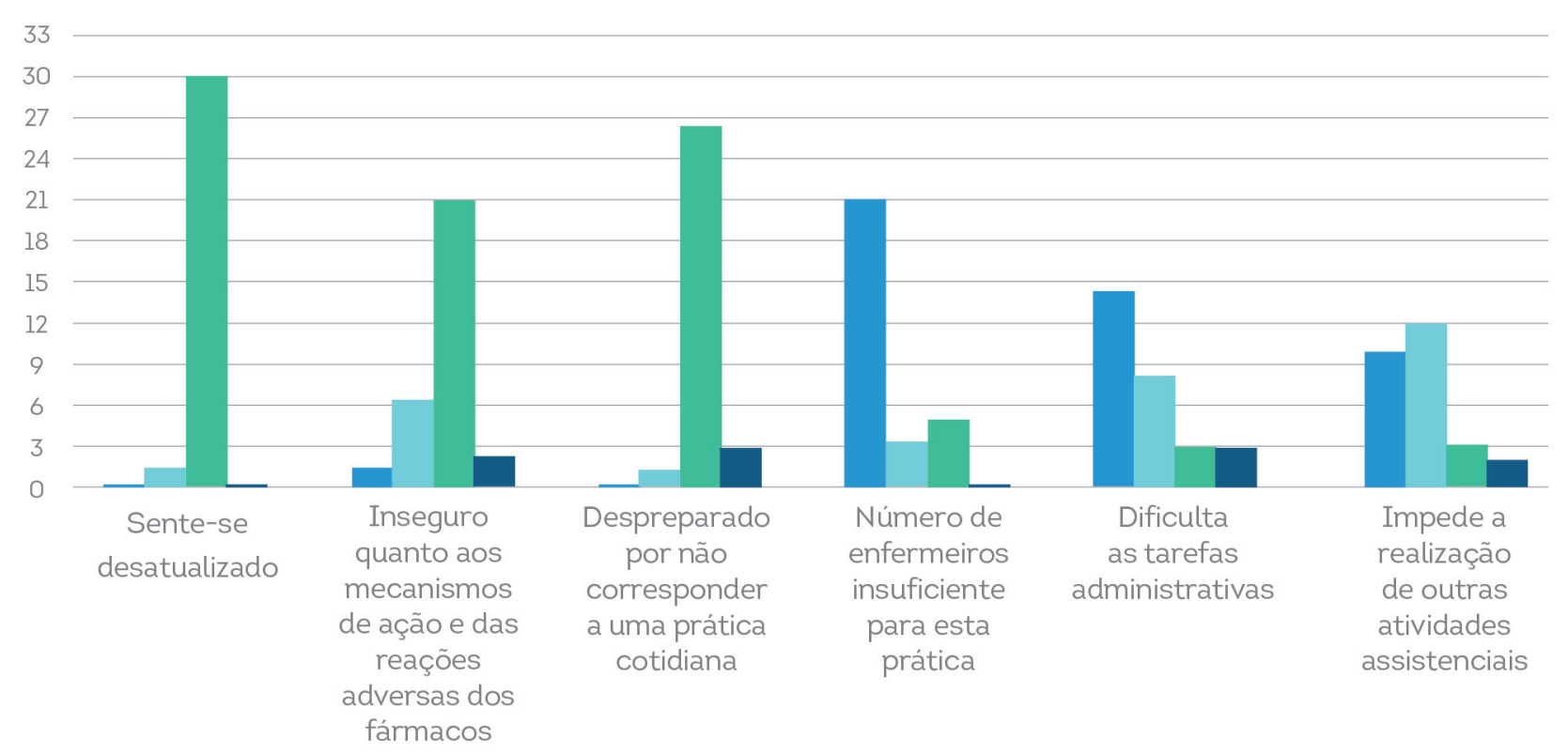

Gráfico 3: Potencialidades e fragilidades na inserção do enfermeiro no processo da prática da administração medicamentosa, Chapecó/SC (2014).

Predominam negativas quanto à: desatualização do tema, insegurança quanto a farmacocinética e o despreparo para a execução. Afirma-se insuficiência do quantitativo de enfermeiros impactando nas tarefas administrativas e impedindo a realização de outras atividades assistenciais.

\section{DISCUSSÃO}

$\mathrm{Na}$ qualificação da atuação do enfermeiro no processo da prática da administração de medicamentos surge inicialmente o instigante questionamento sobrequalé defatoacompetência do enfermeiro neste processo no que diz respeito à equipe de enfermagem? As evidências apontam para uma realidade a qual o enfermeiro demonstra de forma explícita que sua atuação no processo da prática de administração medicamentosa é focada exclusivamente na supervisão

Este fato fica mais demarcado, quando em torno de 2/3 (66\%) dos respondentes declara que a supervisão da administração medicamentosa realizada pelos profissionais de nível superior, somente é implementada quando possivel, permitindo questionar quais seriam os parâmetros desta possibilidade? Neste sentido, se considera inaceitável que o enfermeiro se abstenha de uma responsabilidade cujas repercussões possam se caracterizar como deletérias ao usuário de um serviço de saúde hospitalar.

Ainda, destaca-se a questão da competência da equipe de técnicos/ auxiliares de enfermagem para a administração de medicamentos, sendo que no aparecimento desta dúvida, a prática segura dos cuidados de enfermagem não se torna condizente. $\mathrm{O}$ processo da administração de medicamentos é complexo, no qual a equipe de enfermagem acumula, dentre outros aspectos relacionados a este contexto, a responsabilidade do preparo e administração dos medicamentos, sob supervisão do enfermeiro, com seguimento direto dos efeitos sobre o usuário. Essa prática está amparada pelo Decreto 94.406/87 que regulamenta a lei do exercício da enfermagem, a qual é de responsabilidade do profissional enfermeiro, mesmo que seja executada por outro membro da equipe de enfermagem ${ }^{(7)}$, bem como pelo decreto 94.406/97 
o qual dispõe sobre a obrigatoriedade da supervisão do enfermeiro nas atividades da enfermagem ${ }^{(8)}$.

Ademais, é importante destacar que a comunicação entre a equipe, enfermeiros e técnicos de enfermagem, além das questões operacionais e ambientais, é primordial para a efetividade do processo de administração de medicamentos. Sendo assim, existe a necessidade, por parte do enfermeiro, de uma supervisão criteriosa da técnica executada pela equipe de enfermagem durante o preparo e administração dos medicamentos, assegurando uma comunicação eficiente, êxito no tratamento, prevenindo com isso riscos maiores ao usuário e evitando maiores gastos hospitalares ${ }^{(9)}$.

É importante ressaltar que os técnicos/auxiliares de enfermagem devem possuir sim competência ao desenvolvimento da administração medicamentosa. Entretanto, esta não exime a imprescindivel participação do enfermeiro, seja através da supervisão do processo, e até mesmo por meio da educação no âmbito da equipe de enfermagem.

Segundo $\circ$ Decreto $\mathrm{n}$ 은 94.406/87, artigo 11 inciso III compete ao técnico de enfermagem ministrar medicamentos e o artigo 13 do referido decreto condiciona a efetivação desta atividade, mesmo que realizada pelo técnico, somente sob a orientação, supervisão e direção do enfermeiro(10).
“Dessa forma, o conhecimento em saúde está em permanente aperfeiçoamento especialmente em virtude de estudos e pesquisas
clínicas".
Há necessidade de educação continuada sobre farmacologia para os enfermeiros, visto que esta prática aumentaria a confiança na administração e gerenciamento dos enfermeiros na prática medicamentosa. O conhecimento insuficiente é um dos fatores que mais contribui para erros de medicação(11-13). Neste sentido, estudo quase-experimental ${ }^{(13)}$ ao realizar capacitação de técnicos de enfermagem para a administração de broncodilatadores em pacientes mecanicamente ventilados, constatou que a prática efetuada anteriormente era inadequada o que inviabilizava a adequada deposição do fármaco nas vias aéreas inferiores. A assertiva é que o enfermeiro é responsável técnico e ético pelos cuidados de enfermagem prestados ao paciente. Logo, deve interessar a este profissional trabalhar com uma equipe de técnicos de enfermagem competentes e cientes da necessidade de prestar um cuidado com qualidade e efetividade.
$\mathrm{Na}$ sequência, analisaram-se as potencialidades e fragilidades para a atuação efetiva do enfermeiro na prática da administração medicamentosa. Assim sendo, potencialmente o enfermeiro participante do estudo sentese atualizado em relação à temática em foco, tem segurança quanto ao conhecimento que possui sobre os mecanismos de ação dos fármacos, os seus efeitos colaterais além de sentir-se preparado para a sua execução a despeito de não corresponder uma atividade cotidiana quanto à práxis em si.

Dessa forma, o conhecimento em saúde está em permanente aperfeiçoamento especialmente em virtude de estudos e pesquisas clínicas. No que diz respeito aos fármacos, a educação em saúde tem vital importância para a prestação de um cuidado de qualidade, visto que a indústria farmacológica cresce vertiginosamente, exigindo conhecimento e atenção por parte dos profissionais, especialmente da equipe de enfermagem ${ }^{(13-15)}$.

Quanto às fragilidades, os aspectos salientados preponderantemente designam a problemática do quantitativo de enfermeiros nas unidades de trabalho, a qual acaba por inviabilizar uma participação mais efetiva do enfermeiro no processo da administração medicamentosa. Além disso, observou-se, através das respostas dos participantes, o fato de que esta atuação viria a comprometer as atribuições administrativas pelas quais 0 enfermeiro deve responsabilizarse, associado às demais

atividades assistenciais.

Portanto, condições apropriadas de trabalho são determinantes para um processo de trabalho eficaz, dentre eles um dimensionamento adequado de pessoal por turno, equipamentos, materiais e medicamentos em quantidade e qualidade compativeis com a assistência necessária às pessoas. As más condições de trabalho além de prejudiciais à existência de um processo de trabalho desejável, para a produção da assistência de enfermagem com qualidade, podem ocasionar consequências negativas ao cliente, ao trabalhador, à sua equipe e à instituição de saúde(16).

Em contrapartida, quanto aos obstáculos administrativos e assistenciais que possam ocorrer mediante a responsabilidade com o processo da prática medicamentosa, poderíamos questionar se esta prática 
não se inclui também no rol de atividades da enfermagem, que, por sua natureza, desencadeiam ações de caráter administrativo e assistencial, visando sua efetivação. Com isso, poder-se-ia supor que o cuidado de enfermagem, administrar medicamentos aos usuários de um serviço de saúde, por vezes, para os enfermeiros, assume em uma escala hierárquica um status de menor importância, que lhe permita prescindir sua atuação enquanto supervisor, se comparado a outros procedimentos privativos ao enfermeiro. Paradoxalmente, a este procedimento, facilmente, facultar-se-ia o destaque de ser a mais importante atribuição da equipe de enfermagem.

\section{CONSIDERAÇÕES FINAIS}

Observou-se por meio do estudo que o comprometimento do enfermeiro com a administração medicamentosa é inconsistente, isto é, podendo ser emitida como presente apenas por um caráter legal e ético. Contudo, as condições de trabalho as quais este profissional é exposto, poderiam, de alguma forma, justificar esta inconsistência, pois declara a insuficiência no quantitativo de enfermeiros para a execução de ações denominadas como administrativas e que são cobradas aos mesmos em seu cotidiano assistencial.

Contraditoriamente, os profissionais sentem-se atualizados, conhecedores dos pressupostos científicos referentes a esta prática, assim como capazes de executá-la. Entretanto, esta é uma condição que impacta com a efetiva atuação observada, visto que, no desenvolvimento da expertise é condição "sine qua nom" envolvimento diante de uma prática.

Diante desta realidade, seria oportuno que os gestores do serviço analisassem, de forma criteriosa, o correto dimensionamento de pessoal de enfermagem, considerando, para tal, a legislação que pauta sobre esta questão, visando minimizar esta problemática. Assim, recomendam-se estratégias desenvolvidas pela educação permanente das instituições hospitalares, permitindo a reflexão crítica do profissional enfermeiro, acerca de seu papel diante da prática medicamentosa, avançando de um envolvimento legal, para um envolvimento ético, assegurando, ao usuário, o seu direito de obter o melhor atendimento em saúde em todos os cuidados executados pela enfermagem.
REFERÊNCIAS

1. Cortez EA, Soares GRS, Silva ICM, Carmo TG, Carmo TnG. Preparo e administração venosa de medicamentos e soros sob a ótica da Resolução COFEN n³11/07. Acta Paul Enferm. 2010; 23(6): 843-51.

2. Potter PA. PerryAG. Fundamentos de Enfermagem. 8 ed.Rio de Janeiro: Elsevier, 2013

3. Folkmann L, Rankin J. Nurses' medication work: what do nurses know? Journal of Clinical Nursing. Nov 2010; 19; 3218- 26.

4. Hemingway S, Baxter H, Smith G, Burgess-Dawson R, Dewhirst k. Collaboratively planning for medicines administration competency: a survey evaluation. J Nurs Manag. 2011;19(3):366-76.

5.Westbrook JI Woods A, Rob MI, Dunsnuir WTM, Day RO. Association of interruptions with an increased risk and severity of medication administration errors. Arch intern med. 2010; 170 (8); 683-90.

6. Polit DF, Beck CT. Fundamentos de pesquisa em enfermagem avaliação de evidências para a prática da enfermagem. Porto Alegre: Artmed; 2011.

7. Wei L. Medication communication between nurses and patients during nursing handovers on medical wards: A critical ethnographic study. (Report). Journal of Nursing Studies. Agosto 2012; 49(8); 941-53.

8. COREN (João Pessoa). Sobre a obrigatoriedade da supervisão do Enfermeiro nas atividades da Enfermagem. 2013.

9. Ramos DC, Caetano JÁ, Nascimento JC, Freitas BNG, Teles LMR, Miranda MDC. Avaliação do desempenho da equipe de enfermagem na administração de fármacos via intubação gastrointestinal. Revista Electronica de Enfermagem. [Internet]. 2012 jul/set; 14(3):570-8.

10. Vargas MAO, Luz AMHL. Práticas seguras do/no cuidado de enfermagem no contexto hospitalar: é preciso pensar sobre isso e aquilo. Enferm. Foco. Maio 2010; 1(1): 23-7.

11. Lu MC, Yu S, Chen IJ, Wang KW, Wu HF, Tang FI. Nurses' knowledge of high-alert medications: a randomized controlled trial. Enfermeira. Educ Hoje . 2013; 33 (1); 24-30.

12. Ndosi ME, Newell R. Nurses' knowledge of pharmacology behind drugs they commonly administer. J Clin Nurs. Fev 2009; 18 (4); 570-80.

13. Vargas MAO, Teixeira C, Zanchin F, Ghiot A, Paul K, Schoeller SD. Capacitação dos técnicos de enfermagem para as melhores práticas no uso de broncodilatadores em pacientes mecanicamente ventilados. Texto contexto - enferm. [online]. 2012;21(3):505-12.

14. Calabres N, Louzada SS, Stang F. Administrar e humanizar no hospital. Revista da Faculdade Cenecista. 2008:68-75.

15. Souza EJ. O conhecimento dos profissionais de enfermagem na administração de medicamentos por via intramuscular no local de Hochstetter. Trabalho de Conclusão de Curso (Graduação em Enfermagem)-Universidade do Oeste de Santa Catarina; 2010.

16. Ribeiro J, Rocha LP, Pimpão FD, Porto AR, Thofern MB, et.al. Implicações do ambiente no desenvolvimento do processo de trabalho da enfermagem: uma revisão integrativa. Enfermeria Global. 2012;27:38696. 